\title{
Automated External Defibrillators and Survival After In-Hospital Cardiac Arrest
}

\author{
Paul S. Chan, MD MSc ${ }^{\dagger}$, Harlan M. Krumholz, MD SM ${ }^{@}$, John A. Spertus, MD MPH ${ }^{\dagger}$, Philip \\ G. Jones, MS ${ }^{\dagger}$, Peter Cram, MD\#, Robert A. Berg, MD", Mary Ann Peberdy, MDף, Vinay \\ Nadkarni, MD\|, Mary E. Mancini, RN PhD\&, Brahmajee K. Nallamothu, MD MPH ${ }^{\S}$, and for the \\ American Heart Association National Registry of CPR (NRCPR) Investigators \\ †Saint Luke's Mid America Heart Institute, Kansas City, MO \\ @Section of Cardiovascular Medicine and the Robert Wood Johnson Clinical Scholars Program, \\ Department of Medicine; and the Section of Health Policy and Administration, Department of \\ Epidemiology and Public Health, Yale University School of Medicine; and the Center for \\ Outcomes Research and Evaluation; Yale New Haven Hospital, New Haven, CT \\ \#University of lowa, lowa City, IA \\ "The Children's Hospital of Philadelphia, Philadelphia, PA \\ IVirginia Commonwealth University, Richmond, VA \\ \&The University of Texas at Arlington College of Nursing, Arlington, Texas \\ §The VA Ann Arbor Health Services Research \& Development Center of Excellence and the \\ University of Michigan Division of Cardiovascular Medicine, Ann Arbor, Ml
}

\section{Abstract}

Context-Automated external defibrillators (AEDs) improve survival from out-of-hospital cardiac arrests, but data on their effectiveness in hospitalized patients are limited.

Objective-To evaluate the association of AED use and survival for in-hospital cardiac arrest.

Design, Setting, Patients-Cohort study of 11,695 hospitalized patients with cardiac arrests between January 1, 2000 and August 26, 2008 at 204 hospitals following the introduction of AEDs on general hospital wards.

Main Outcome Measure-Survival to hospital discharge by AED use, using multivariable hierarchical regression analyses to adjust for patient factors and hospital site.

Send correspondence and reprint requests to: Paul Chan, MD, MSc, Mid America Heart Institute, $5^{\text {th }}$ Floor, 4401 Wornall Road, Kansas City, MO 64111, Phone: (816) 699-3409, Fax: (816) 932-5613, Attn: Paul Chan, pchan @ cc-pc.com.

Disclosures:

None of the authors report any potential conflicts of interest or disclosures.

Authorship: Dr. Chan had full access to all of the data in the study, and takes responsibility for the integrity of the data and the accuracy of the data analysis.

Study Concept and design: Chan, Nallamothu

Acquisition of Data: Chan

Statistical Analysis: Jones

Analysis and interpretation of data: Chan, Krumholz, Spertus, Jones, Cram, Berg, Peberdy, Nadkarni, Mancini, Nallamothu

Drafting of the manuscript: Chan, Nallamothu

Critical revision of the manuscript for important intellectual content: Chan, Krumholz, Spertus, Jones, Cram, Berg, Peberdy, Nadkarni,

Mancini, Nallamothu

Study Supervision: Chan, Nallamothu 
Results-Of 11,695 patients, 9616 (82.2\%) had non-shockable rhythms (asystole and pulseless electrical activity) and 2079 (17.8\%) had shockable rhythms (ventricular fibrillation and pulseless ventricular tachycardia). AEDs were used in 4515 (38.6\%) patients. Overall, 2117 (18.1\%) patients survived to hospital discharge. Within the entire study population, AED use was associated with a lower rate of survival after in-hospital cardiac arrest compared with no AED use ( $16.3 \%$ vs. $19.3 \%$; adjusted rate ratio (RR), 0.85 ; $95 \%$ confidence interval (CI), 0.78-0.92; $\mathrm{P}<0.001)$. Among cardiac arrests due to non-shockable rhythms, AED use was associated with lower survival (10.4\% vs. $15.4 \%$; adjusted RR, 0.74 ; 95\% CI, 0.65-0.83; P<.001). In contrast, for cardiac arrests due to shockable rhythms, AED use was not associated with survival (38.4\% vs. $39.8 \%$; adjusted RR, 1.00; 95\% CI, 0.88-1.13; $\mathrm{P}=0.99$ ). These patterns were consistently observed in both monitored and non-monitored hospital units where AEDs were used, after matching patients to the individual units in each hospital where the cardiac arrest occurred, and with a propensity score analysis.

Conclusion-Use of AEDs in hospitalized patients with cardiac arrest is not associated with improved survival.

\section{Introduction}

Use of automated external defibrillators (AEDs) has been proposed as a strategy to reduce times to defibrillation and improve survival from cardiac arrests that occur in the hospital setting. ${ }^{1,2}$ However, current evidence to support the use of AEDs in hospitals has been mixed and limited to single-center studies. ${ }^{3,4}$ While some studies have shown that AEDs improve survival for out-of-hospital cardiac arrests occurring in certain public locations in which $45 \%$ to $71 \%$ of cases are treatable with defibrillation, ${ }^{5-7}$ these devices may be less effective or potentially harmful when used in hospitals where only 1 in 5 hospitalized patients have initial cardiac arrest rhythms that respond to defibrillation. ${ }^{8}$ Furthermore, the use of AEDs requires manual application of defibrillator pads and automated rhythm analysis to determine whether a cardiac arrest rhythm is "shockable" or not. Both steps may lead to interruptions in continuous chest compressions that are delivered during the critical first minutes of acute resuscitation and adversely affect survival. Before endorsing their widespread dissemination in hospitals, it therefore becomes critical to demonstrate that AED use improves survival.

Accordingly, we used data from the National Registry of Cardiopulmonary Resuscitation (NRCPR) to evaluate the association of AED use and survival after an in-hospital cardiac arrest. The large size of the NRCPR permits a more detailed analysis of AED use stratified by the initial cardiac arrest rhythm and location of the cardiac arrest - both of which are factors that may influence its effectiveness in hospitalized patients.

\section{Methods}

\section{Study Design}

The NRCPR is a large, prospective, quality improvement registry of in-hospital cardiac arrests, and its design has been previously described in detail. ${ }^{8}$ Briefly, all patients with cardiac arrest (defined as the absence of a palpable central pulse, apnea, and unresponsiveness) and without Do-Not-Resuscitation (DNR) orders are identified and enrolled by specially-trained quality improvement personnel. Cases are identified by multiple methods, including centralized collection of cardiac arrest flow sheets, reviews of hospital paging system logs, and routine checks of code carts, pharmacy tracer drug records, and hospital billing charges for use of resuscitation medications. ${ }^{8}$ 
The NRCPR uses standardized Utstein-style definitions, which are a template of uniform reporting guidelines developed by international experts, for defining patient variables and outcomes to facilitate uniform reporting across hospitals. ${ }^{9}, 10$ Data accuracy is ensured by rigorous certification of hospital staff and use of standardized software with data checks for data completeness and accuracy. ${ }^{11}$ For this study, the Institutional Review Board (IRB) of the Mid America Heart Institute waived the requirement for informed consent.

\section{Study Population}

Our cohort was derived from 550 acute-care hospitals that provided at least 6 months of data to the NRCPR between January 1, 2000 and August 26, 2008. Within these hospitals, we identified 110,132 patients 18 years of age or older with an index pulseless in-hospital cardiac arrest (Figure 1). Given the focus of our analysis, we included only those patients with cardiac arrests occurring in general hospital wards where AEDs are most likely to be deployed. After these exclusions, there remained 31,838 cardiac arrests occurring in general hospital wards.

Next, we restricted our analyses to those hospitals where an AED was used in at least one cardiac arrest, leading us to exclude an additional 14,185 cardiac arrests from 346 hospitals without AEDs. Because hospitals in our sample introduced AEDs at varying time points between 2000 and 2008, we only included cardiac arrest cases submitted after the first date documenting AED use within respective monitored and non-monitored units in each hospital (5958 arrests excluded) to ensure that comparisons involved contemporary patients in the same facility. The final study sample included 11,695 patients from 204 hospitals.

\section{AED and Outcomes}

The key independent variable was whether an AED was used during the initial assessment of a patient to determine the cardiac arrest rhythm and appropriateness of defibrillation. As such, AED use included either stand-alone, automated devices that are commercially available or manual defibrillators placed in AED mode.

The primary outcome was survival to hospital discharge. In addition, we examined as secondary outcomes return of spontaneous circulation (ROSC) for at least 20 minutes during the acute resuscitation, survival at 24 hours, and neurological status among those surviving to hospital discharge. Neurological status was assessed using previously-developed cerebral performance categories (CPC), ${ }^{12}$ and we classified patients as being without (CPC score of 1) or with (CPC scores of >1) major neurological disability. ${ }^{13}$ Data were missing for survival at 24 hours in $74(0.6 \%)$ patients and for CPC scores in $267(12.6 \%)$ survivors, and rates of missing data for these outcomes were similar $(\mathrm{P}>0.10)$ in the AED and non-AED groups. Otherwise, data for all other outcomes, as well as patient characteristics, were complete.

\section{Statistical Analyses}

Patients were classified by their initial cardiac arrest rhythm (ventricular fibrillation, pulseless ventricular tachycardia, asystole, or pulseless electrical activity). Baseline differences between patients in whom AEDs were and were not used were evaluated using $X^{2}$ tests for categorical variables and Student's t-tests for continuous variables. In addition, Wilcoxon rank-sum tests were employed for comparisons of time-related variables with non-normal distributions.

Multivariable hierarchical regression models were used to assess the relationship between AED use and survival. We employed 2-level hierarchical models to adjust for clustering of outcomes within hospitals, with individual hospitals modeled as random effects and other 
patient and hospital characteristics modeled as fixed effects within each hospital. ${ }^{14}$ This approach allowed us to control for measured and unmeasured between-hospital confounding, as the use of hierarchical models ensured that patients assessed with AEDs were only compared with patients not assessed with AEDs from the same hospital. Within each hospital, we additionally controlled for age, sex, race (white, black, other), monitoring status (monitored, non-monitored), initial cardiac arrest rhythm, calendar year of event, time (work hours: $7 \mathrm{am}$ to $10: 59 \mathrm{pm}$ vs. after hours: $11 \mathrm{pm}$ to 6:59am) and day (weekday vs. weekend) of cardiac arrest, ${ }^{11}$ use of a hospital-wide cardiopulmonary arrest alert, comorbidities or medical conditions present prior to cardiac arrest (congestive heart failure, myocardial infarction, or diabetes mellitus; renal, hepatic, or respiratory insufficiency; baseline evidence of motor, cognitive, or functional deficits [CNS depression]; acute stroke; acute non-stroke neurologic disorder; pneumonia; hypotension; sepsis; major trauma; metabolic or electrolyte abnormality; and metastatic or hematologic malignancy), and the proportion of cardiac arrests evaluated by an AED at each hospital. Because survival rates exceeded $10 \%$, we utilized modified Poisson regression models with robust variance estimates at all steps to directly estimate rate ratios (RRs), as odds ratios obtained from logistic regression may overestimate survival differences when the frequency of the outcome of interest is not rare (e.g., $>10 \%) .{ }^{15,16}$

The association between AED use and survival was further examined after categorizing patients based on the responsiveness of the initial cardiac arrest rhythm to defibrillation. "Non-shockable" rhythms were asystole and pulseless electrical activity, while "shockable" rhythms were ventricular fibrillation and pulseless ventricular tachycardia. In analyses of arrests due to ventricular fibrillation and pulseless ventricular tachycardia, models also adjusted for time to defibrillation. Based on prior work, time to defibrillation was calculated as the time from initial recognition of the cardiac arrest to the time of first attempted defibrillation and was examined as both a continuous variable and as delayed ( $>2$ minutes) vs. not delayed ( $\mathcal{2}$ minutes). ${ }^{13,17}$ Furthermore, we examined for an interaction between AED use and monitoring status for the outcome of survival to discharge.

As a sensitivity analysis, we repeated our analyses $f$ or the outcome of survival to discharge after further matching patients within each hospital by (1) the specific hospital ward in which cardiac arrests occurred and (2) the hospital ward and calendar year of the arrest, comparing outcomes in both instances using Cochran-Mantel-Haenszel tests that were stratified by matched sets. ${ }^{18}$ We also modeled time as 6-month periods after AED implementation in each hospital within our hierarchical models and tested for a temporal trend for survival to discharge after AED implementation. These analyses allowed us to additionally control for variation in the quality of care that may exist across wards within each hospital and to evaluate whether there were temporal trends in the association between AED use and survival.

Finally, to assess the robustness of our findings, we performed a propensity score analysis to compare survival between the AED groups. ${ }^{19-21}$ Separate propensity score models for AED use were constructed for patients with cardiac arrests due to shockable (C-statistic of 0.81) and non-shockable rhythms (C-statistic of 0.82). All variables used in the hierarchical models described above, as well as the hospital center, were included as covariates in multivariable logistic regression models to derive the propensity scores. Then, within each rhythm group, we conducted a 1:1 propensity score match between patients assessed and not assessed with AEDs, using an algorithm match with a caliper width no greater than 0.2 times the standard deviation of the logit of the propensity score. ${ }^{22}$ This resulted in a successful match for 3004 patients in whom AEDs were used. We confirmed that AED and non-AED patients were well-balanced in covariates after propensity score matching by ensuring that standardized differences between the AED groups for each covariate were $<10 .{ }^{23}$ The 
association between AED use and survival to discharge was then assessed using the Cochran-Mantel-Haenszel test to ensure comparisons between matched patients. ${ }^{18}$

We also constructed multivariable hierarchical models to examine the association between AED use and the secondary outcomes of ROSC, survival at 24 hours, and major neurological disability among those surviving to discharge. Finally, to further explore our findings, we examined the association of AED use on time to defibrillation, number of defibrillations administered, and duration of the acute resuscitation in those patients with ROSC. Resuscitation time was calculated as the time from initial recognition of the cardiac arrest to the time of ROSC.

As AEDs are expected to influence survival only in cardiac arrests due to ventricular fibrillation or pulseless ventricular tachycardia, we conducted a retrospective power analysis for these rhythms. Given the rate of survival to discharge in the non-AED group, our patient sample, and a 2-sided significance level of 0.05 , our study had $95 \%$ statistical power to detect a $20 \%$ increase, and $78 \%$ statistical power to detect a $15 \%$ increase, in survival with AED use.

All subgroup and secondary analyses were pre-specified. Goodness of fit (HosmerLemeshow statistic) and calibration plots were confirmed for each of the models. For each analysis, the null hypothesis was evaluated at a two-sided significance level of 0.05 , with 95\% confidence intervals (CIs) calculated using robust standard errors. All analyses were performed with SAS 9.2 (SAS Institute, Cary, NC) and R version 2.10.0. ${ }^{24}$

\section{Results}

We identified 11,695 patients with cardiac arrests at 204 hospitals after the introduction of AEDs. Of these, 2079 (17.8\%) had shockable rhythms, such as ventricular fibrillation or pulseless ventricular tachycardia, and 9616 (82.2\%) had non-shockable rhythms, such as asystole or pulseless electrical activity. AEDs were used to assess initial rhythm in 4515 (38.6\%) patients. Tables 1 and 2 display patient, arrest, and hospital characteristics according to whether an AED was used or not. There were no differences by age or sex, although there was a slightly higher rate of AED use in black patients. AED use also was more likely in non-monitored hospital wards and for cardiac arrests due to pulseless ventricular tachycardia or ventricular fibrillation. Importantly, there were no differences in rates of AED use for the majority of other clinical factors.

\section{Survival to Discharge}

Overall, $2117(18.1 \%)$ patients survived to hospital discharge. Within the entire study population, the rate of survival to hospital discharge was $16.3 \%$ (734/4515; 95\% CI: $15.2 \%$ to $17.4 \%$ ) among patients in whom AEDs were used and 19.3\% (1383/7180; 95\% CI: $18.4 \%$ to $20.2 \%$ ) among patients in whom AEDs were not used. After multivariable adjustment for hospital site and clinical characteristics, AED use was associated with a lower rate of survival (adjusted rate ratio [RR], 0.85; 95\% confidence interval [CI] 0.78 to $0.92 ; \mathrm{P}<0.001$ ). The lower adjusted survival associated with AED use was seen regardless of whether the cardiac arrest occurred in a monitored or non-monitored hospital ward ( $\mathrm{P}$ for interaction of 0.51) (Table 3).

The association between AED use and survival to discharge, however, differed by the initial cardiac arrest rhythm (P for interaction of 0.001) (see Table 3). Among the 9616 cardiac arrests due to non-shockable rhythms, such as asystole or pulseless electrical activity, AED use was associated with lower in-hospital survival (10.4\% [95\% CI: $9.4 \%$ to $11.4 \%$ ] for AED use, $15.4 \%$ [95\% CI: $14.5 \%$ to $16.4 \%$ ] for no AED use; adjusted RR, $0.74 ; 95 \%$ CI, 
0.65 to $0.83 ; \mathrm{P}<.001$ ). In contrast, for the 2079 cardiac arrests due to shockable rhythms, such as ventricular fibrillation or pulseless ventricular tachycardia, there was no association between AED use and in-hospital survival (38.4\% [95\% CI: 35.3\% to 41.6\%] for AED use, $39.8 \%$ [ $95 \%$ CI:36.9\% to $42.7 \%$ ] for no AED use; adjusted RR, 1.00; 95\% CI, 0.88 to 1.13; $\mathrm{P}=0.99)$.

These relationships between AED use and survival were consistent in monitored and nonmonitored hospital units for each rhythm type ( $\mathrm{P}$ for interaction between AED use and monitoring status for each rhythm type $>0.10$ ). Our results remained essentially unchanged when we repeated these analyses (1) after matching 7027 (60.1\%) patients to the individual unit in the hospital wh ere the arrest occurred and additionally by calendar year (eTable 1) and (2) when using a matched propensity score analysis (eTable 2). In addition, we examined whether our results differed over time after AED implementation and found no evidence for an interaction ( $\mathrm{P}$ for interaction of 0.91 ) between the first and subsequent years of implementation (eTable 3 ) or for a temporal trend when time was modeled as a continuous variable ( $\mathrm{P}$ for trend of 0.59 ).

Finally, among those surviving to discharge, AED use was not associated with major neurological disability. This finding was consistent for the entire cohort and when stratified by either rhythm type or monitoring status (Table 4).

\section{Secondary Outcomes}

For the majority of patients who had cardiac arrests due to asystole or pulseless electrical activity, rates of ROSC were similar with and without AED use (Table 4). However, AED use was associated with a lower rate of survival at 24 hours after cardiac arrest $(28.0 \%$ [95\% CI: $26.5 \%$ to $29.5 \%$ ] for AED use, $33.8 \%$ [95\% CI: $32.6 \%$ to $35.0 \%$ ] for no AED use, adjusted RR, $0.89,95 \%$ CI, 0.83 to 0.95 ; $\mathrm{P}<.001$ ). Importantly, among patients with ROSC, AED use was associated with longer periods of cardiopulmonary resuscitation prior to restoration of pulse among patients with asystole or pulseless electrical activity (for AED use, median of 15 minutes [inter-quartile range, 9-24]; for no AED use, median of 14 minutes [inter-quartile range, 7-22]; $\mathrm{P}<.001$ ) (eTable 4).

For cardiac arrests due to ventricular fibrillation or pulseless ventricular tachycardia, rates of ROSC and survival at 24 hours were similar with and without AED use (see Table 4). AED use was not associated with shorter times to defibrillation and, among those with ROSC, was not associated with shorter cardiopulmonary resuscitation times or fewer administered defibrillations (eTable 4).

\section{Discussion}

We found that AED use was not associated with improved survival to discharge in hospitalized patients with cardiac arrest. AED use was associated with a lower rate of survival in cardiac arrests due to non-shockable rhythms, such as asystole or pulseless electrical activity. Importantly, such events accounted for more than 4 out of 5 cardiac arrests in the hospitalized setting. For cardiac arrests due to shockable rhythms, such as pulseless ventricular tachycardia or ventricular fibrillation, AED use was not linked to improved survival or shorter times to defibrillation. Our findings therefore do not support the routine use of AEDs for in-hospital cardiac arrests.

Prior observational studies on AED use in hospitals have yielded conflicting results. One study found that survival improved from $4.9 \%$ to $12.8 \%$ ( $\mathrm{P}=0.001)$ after implementation of an AED program within a hospital, ${ }^{3}$ while a second study found no significant association between AED use and survival (18\% vs. $23 \%$; $\mathrm{P}=0.09) .{ }^{4}$ Both studies were limited due to 
small samples. Using a much larger sample of patients at more than 200 hospitals, we were able to evaluate the association of AED use and survival based on the initial cardiac arrest rhythm as well as in both monitored and non-monitored hospital units.

On the surface, our results may appear surprising since AEDs have been shown to improve survival for witnessed out-of-hospital cardiac arrests in public locations. ${ }^{5-7}$ However, our results may differ substantially from those investigations due to differences in the initial cardiac arrest rhythm. In the out-of-hospital setting, $45 \% 6$ to $71 \%^{7}$ of initial arrest rhythms in certain public locations are due to pulseless ventricular tachycardia or ventricular fibrillation. In contrast, only 18\% of our study's in-hospital cardiac arrests were due to these shockable rhythms. This premise is further supported by the results of the Home Use of Automated External Defibrillators for Sudden Cardiac Arrest Trial. Although that trial evaluated the impact of AEDs in out-of-hospital cardiac arrests, only $14 \%$ of arrests were witnessed and treatable by an AED resulting in no effect of these devices on survival. ${ }^{25}$

Of particular concern was our finding that AED use was associated with lower survival in cardiac arrests due to asystole or pulseless electrical activity. The long time period required for using an AED to assess initial cardiac arrest rhythm may provide a mechanism for this finding. Manual application of defibrillator pads and initial rhythm analysis with an AED have been shown to take between 46 and 52 seconds. ${ }^{26,} 27$ Therefore, the time required to use an AED to assess these non-shockable cardiac arrest rhythms may lead to longer interruptions of continuous chest compressions during the first few minutes of resuscitation, when effective cardiac perfusion to vital organs is most critical. ${ }^{28-30}$

Indeed, we found that, among patients surviving the acute resuscitation, AED use was associated with longer resuscitation periods to achieve ROSC for asystole and pulseless electrical activity - perhaps owing to longer periods without chest compressions during the initial minutes - and lower survival as early as 24 hours after cardiac arrest. Because the magnitude of survival difference was apparent within 24 hours from cardiac arrest, the lower survival associated with AED use for cardiac arrests due to these rhythms was likely attributable to factors occurring during acute resuscitation (e.g., longer resuscitation period to achieve ROSC), rather than post-resuscitation.

Somewhat surprisingly, we did not find a survival benefit with AED use in patients with a cardiac arrest due to pulseless ventricular tachycardia or ventricular fibrillation. In large part, this may be because AED use was not associated with shorter times to defibrillation. While it is possible that the time of arrival of an AED to a patient's bedside was shorter than the time required for a conventional defibrillator, this gain may have been offset by the time required to manually apply defibrillator pads and await automated rhythm analysis. In addition, it has been shown that repeat rhythm assessment with an AED after defibrillation takes, on average, 46-seconds prior to resumption of chest compressions, ${ }^{26,31}$ which is 24 seconds longer than with a conventional defibrillator, thus extending the period without continuous chest compressions for patients who do not initially respond to defibrillation. ${ }^{26}$

It is also possible that AEDs may not be as effective in the initial treatment of ventricular tachyarrhythmias as compared with conventional defibrillators, in which defibrillation energies are manually chosen. Indeed, the Food and Drug Administration recently issued a communication expressing concerns of suboptimal defibrillation energy levels delivered during the initial shock by AEDs. ${ }^{32}$ Finally, suboptimal implementation of an AED program and poor training of hospital staff in the use of AEDs may have attenuated the potential benefits of AEDs for cardiac arrests due to shockable rhythms in our study. Future studies with detailed data on AED use, resuscitation process-of-care times, and hospital-level AED 
implementation are needed to clarify the reasons for the lack of benefit of AEDs in these rhythms.

Despite a lack of data on their potential impact, hospitals have increasingly adopted the use of AEDs in patient areas in response to local and national efforts to improve defibrillation time and resuscitation survival. Between 2003 and 2008, over 50,000 AED units were sold to U.S. hospitals, and marketing reports project annual sales growth of $9 \%$ to $12 \%$ over the next 5 years. ${ }^{33}$ In our study sample, we found that $37 \%$ (204/550) of hospitals within the NRCPR had introduced AEDs into their general patient care areas by 2008. In light of our data, national organizations and hospitals may need to reconsider the use of AEDs in general hospital ward units or develop different strategies for employing them.

Our study should be interpreted in the context of the following limitations. First, although data available in the NRCPR allowed us to adjust for a number of key variables that have been linked to survival after cardiac arrest, our study used an observational design and the possibility of residual confounding remains. Despite our use of robust statistical methods, our findings require further confirmation with a randomized controlled trial. Second, the NRCPR did not collect data on the time of arrival of an AED to the patient's bedside, the time required for automated rhythm analysis, and the extent of interruptions of chest compressions. Such data may have been valuable in explaining our findings but are often difficult to obtain or document accurately. Similarly, while we had data on time to defibrillation, there may be inaccuracies in its documentation. ${ }^{34}$ Nevertheless, the primary outcome in this study was survival to discharge, and we examined defibrillation time as an explanatory variable for some of our findings.

Third, the NRCPR did not collect data about the AED implementation strategy at each hospital. This prevented us from better understanding whether our study findings could have been due to a failure of AED implementation, poor training of hospital personnel, or lack of integration with other hospital quality improvement initiatives, such as rapid response teams. Fourth, it is possible that some AEDs were used in manual mode. However, while use of the AED in manual mode may explain our null findings in shockable rhythms, it can not explain worse survival in patients with non-shockable rhythms.

Fifth, we did not have information as to why an AED was or was not used for a given cardiac arrest despite its availability at the institution and even on the individual unit of the hospital. For example, it remains possible that AEDs were more commonly used by lesstrained providers. Finally, the NRCPR is a quality improvement registry that collects cardiac arrest data from a diverse population of U.S. hospitals. Although these facilities represent nearly $15 \%$ of U.S. hospitals with more than 250 beds, the association of AED use and survival may be different in nonparticipating hospitals.

In conclusion, we found that use of AEDs to assess and treat hospitalized patients with cardiac arrests was not associated with improved survival. While randomized controlled trials are needed to confirm these findings, current use of AEDs in hospitalized patients may warrant reconsideration.

\section{Supplementary Material}

Refer to Web version on PubMed Central for supplementary material.

\section{Acknowledgments}

Beside the authors Paul S. Chan, MD; Robert Berg, MD; Mary Mancini, PhD; Vinay Nadkarni, MD; and Mary Ann Peberdy, MD; the American Heart Association National Registry of CPR investigators include as well as: 
1. Emilie Allen, BSN; Parkland Health and Hospital System

2. Scott Braithwaite, MD; New York University School of Medicine

3. Dana Edelson, MD; University of Chicago School of Medicine

4. Brian Eigel, MD; American Heart Association

5. Romer Geocadin, MD; Johns Hopkins School of Medicine

6. Elizabeth Hunt, MD; Johns Hopkins School of Medicine

7. Karl Kern, MD; University of Arizona at Tucson

8. Timothy Mader, MD; Baystate Medical Center/Tufts University School of Medicine

9. Graham Nichol, MD; University of Washington

10. Thomas Noel, MD; Virginia Commonwealth University

11. Joseph Ornato, MD; Virginia Commonwealth University

12. Comilla Sasson, MD; University of Colorado at Denver

13. Tanya Truitt, RN; American Heart Association

14. Samuel Warren, MD; University of Washington

The American Heart Association provided funding for the collection and management of data in the NRCPR and reviewed and approved the final manuscript. However, it had no role in the design and conduct of this study, the analysis or interpretation of the data, or the preparation of this manuscript.

\section{REFERENCES}

1. Saxon LA. Survival after tachyarrhythmic arrest--what are we waiting for? N Engl J Med. 2008; 358(1):77-79. [PubMed: 18172179]

2. 2005 American Heart Association Guidelines for Cardiopulmonary Resuscitation and Emergency Cardiovascular Care. Part 5: Electrical Therapies. Circulation. 2005; 112(24 Suppl):IV35-IV46.

3. Zafari AM, Zarter SK, Heggen V, et al. A program encouraging early defibrillation results in improved in-hospital resuscitation efficacy. J Am Coll Cardiol. 2004; 44(4):846-852. [PubMed: 15312869]

4. Forcina MS, Farhat AY, O'Neil WW, Haines DE. Cardiac arrest survival after implementation of automated external defibrillator technology in the in-hospital setting. Crit Care Med. 2009; 37(4): 1229-1236. [PubMed: 19326574]

5. Weaver WD, Hill D, Fahrenbruch CE, et al. Use of the automatic external defibrillator in the management of out-of-hospital cardiac arrest. N Engl J Med. 1988; 319(11):661-666. [PubMed: 3412383]

6. Hallstrom AP, Ornato JP, Weisfeldt M, et al. Public-access defibrillation and survival after out-ofhospital cardiac arrest. N Engl J Med. 2004; 351(7):637-646. [PubMed: 15306665]

7. Valenzuela TD, Roe DJ, Nichol G, Clark LL, Spaite DW, Hardman RG. Outcomes of rapid defibrillation by security officers after cardiac arrest in casinos. N Engl J Med. 2000; 343(17):12061209. [PubMed: 11071670]

8. Peberdy MA, Kaye W, Ornato JP, et al. Cardiopulmonary resuscitation of adults in the hospital: a report of 14720 cardiac arrests from the National Registry of Cardiopulmonary Resuscitation. Resuscitation. 2003; 58(3):297-308. [PubMed: 12969608]

9. Cummins RO, Chamberlain D, Hazinski MF, et al. Recommended guidelines for reviewing, reporting, and conducting research on in-hospital resuscitation: the in-hospital 'Utstein style'. American Heart Association. Circulation. 1997; 95(8):2213-2239. [PubMed: 9133537]

10. Jacobs I, Nadkarni V, Bahr J, et al. Cardiac arrest and cardiopulmonary resuscitation outcome reports: update and simplification of the Utstein templates for resuscitation registries: a statement for healthcare professionals from a task force of the International Liaison Committee on Resuscitation (American Heart Association, European Resuscitation Council, Australian Resuscitation Council, New Zealand Resuscitation Council, Heart and Stroke Foundation of 
Canada, InterAmerican Heart Foundation, Resuscitation Councils of Southern Africa). Circulation. 2004; 110(21):3385-3397. [PubMed: 15557386]

11. Peberdy MA, Ornato JP, Larkin GL, et al. Survival from in-hospital cardiac arrest during nights and weekends. Jama. 2008; 299(7):785-792. [PubMed: 18285590]

12. Jennett B, Bond M. Assessment of outcome after severe brain damage. Lancet. 1975; 1(7905):480484. [PubMed: 46957]

13. Chan PS, Krumholz HM, Nichol G, Nallamothu BK. Delayed time to defibrillation after inhospital cardiac arrest. N Engl J Med. 2008; 358(1):9-17. [PubMed: 18172170]

14. Goldstein, H. Multilevel Statistical Models. London and New York: Edward Arnold; Wiley; 1995.

15. Greenland S. Model-based estimation of relative risks and other epidemiologic measures in studies of common outcomes and in case-control studies. Am J Epidemiol. 2004; 160(4):301-305. [PubMed: 15286014]

16. Zou G. A modified poisson regression approach to prospective studies with binary data. Am J Epidemiol. 2004; 159(7):702-706. [PubMed: 15033648]

17. Chan PS, Nichol G, Krumholz HM, Spertus JA, Nallamothu BK. Hospital variation in time to defibrillation after in-hospital cardiac arrest. Arch Intern Med. 2009; 169(14):1265-1273. [PubMed: 19636027]

18. Walker, GA. Common Statistical Methods for Clinical Research with SAS® Examples. Second Edition. Cary, N.C.; 2002.

19. D'Agostino RB Jr. Propensity scores in cardiovascular research. Circulation. 2007; 115(17):23402343. [PubMed: 17470708]

20. Rubin DB. Estimating causal effects from large data sets using propensity scores. Ann Intern Med. 1997; 127(8 Pt 2):757-763. [PubMed: 9382394]

21. Rosenbaum PR, Rubin DB. The central role of the propensity score in observational studies for causal effects. Biometrika. 1983; 70:41-55.

22. Rosenbaum PR, Rubin DB. Constructing a control group using multivariate matched sampling methods that incorporate propensity scores. The American Statistician. 1985; 39(1):33-38.

23. Normand ST, Landrum MB, Guadagnoli E, et al. Validating recommendations for coronary angiography following acute myocardial infarction in the elderly: a matched analysis using propensity scores. Journal of clinical epidemiology. 2001; 54(4):387-398. [PubMed: 11297888]

24. R Development Core Team. R: A language and environment for statistical computing. $\mathrm{R}$ Foundation for Statistical Computing V, Austria. 2008 ISBN 3-900051-07-0, URL http://www.Rproject.org.

25. Bardy GH, Lee KL, Mark DB, et al. Home use of automated external defibrillators for sudden cardiac arrest. N Engl J Med. 2008; 358(17):1793-1804. [PubMed: 18381485]

26. Berg RA, Hilwig RW, Kern KB, Sanders AB, Xavier LC, Ewy GA. Automated external defibrillation versus manual defibrillation for prolonged ventricular fibrillation: lethal delays of chest compressions before and after countershocks. Ann Emerg Med. 2003; 42(4):458-467. [PubMed: 14520316]

27. Berg MD, Clark LL, Valenzuela TD, Kern KB, Berg RA. Post-shock chest compression delays with automated external defibrillator use. Resuscitation. 2005; 64(3):287-291. [PubMed: 15733755]

28. Bobrow BJ, Clark LL, Ewy GA, et al. Minimally interrupted cardiac resuscitation by emergency medical services for out-of-hospital cardiac arrest. Jama. 2008; 299(10):1158-1165. [PubMed: 18334691]

29. Kern KB, Hilwig RW, Berg RA, Sanders AB, Ewy GA. Importance of continuous chest compressions during cardiopulmonary resuscitation: improved outcome during a simulated single lay-rescuer scenario. Circulation. 2002; 105(5):645-649. [PubMed: 11827933]

30. Sanders AB, Kern KB, Berg RA, Hilwig RW, Heidenrich J, Ewy GA. Survival and neurologic outcome after cardiopulmonary resuscitation with four different chest compression-ventilation ratios. Ann Emerg Med. 2002; 40(6):553-562. [PubMed: 12447330]

31. van Alem AP, Sanou BT, Koster RW. Interruption of cardiopulmonary resuscitation with the use of the automated external defibrillator in out-of-hospital cardiac arrest. Ann Emerg Med. 2003; 42(4):449-457. [PubMed: 14520315] 
32. Food and Drug Administration; 2009. Energy Levels in External Biphasic Defibrillators: Initial Communication. http://www.fda.gov/MedicalDevices/Safety/AlertsandNotices/ucm189259.htm.

33. Frost and Sullivan. U.S. AED Market: Market Analysis. 2007

34. Kaye W, Mancini ME, Truitt TL. When minutes count--the fallacy of accurate time documentation during in-hospital resuscitation. Resuscitation. 2005; 65(3):285-290. [PubMed: 15919564] 


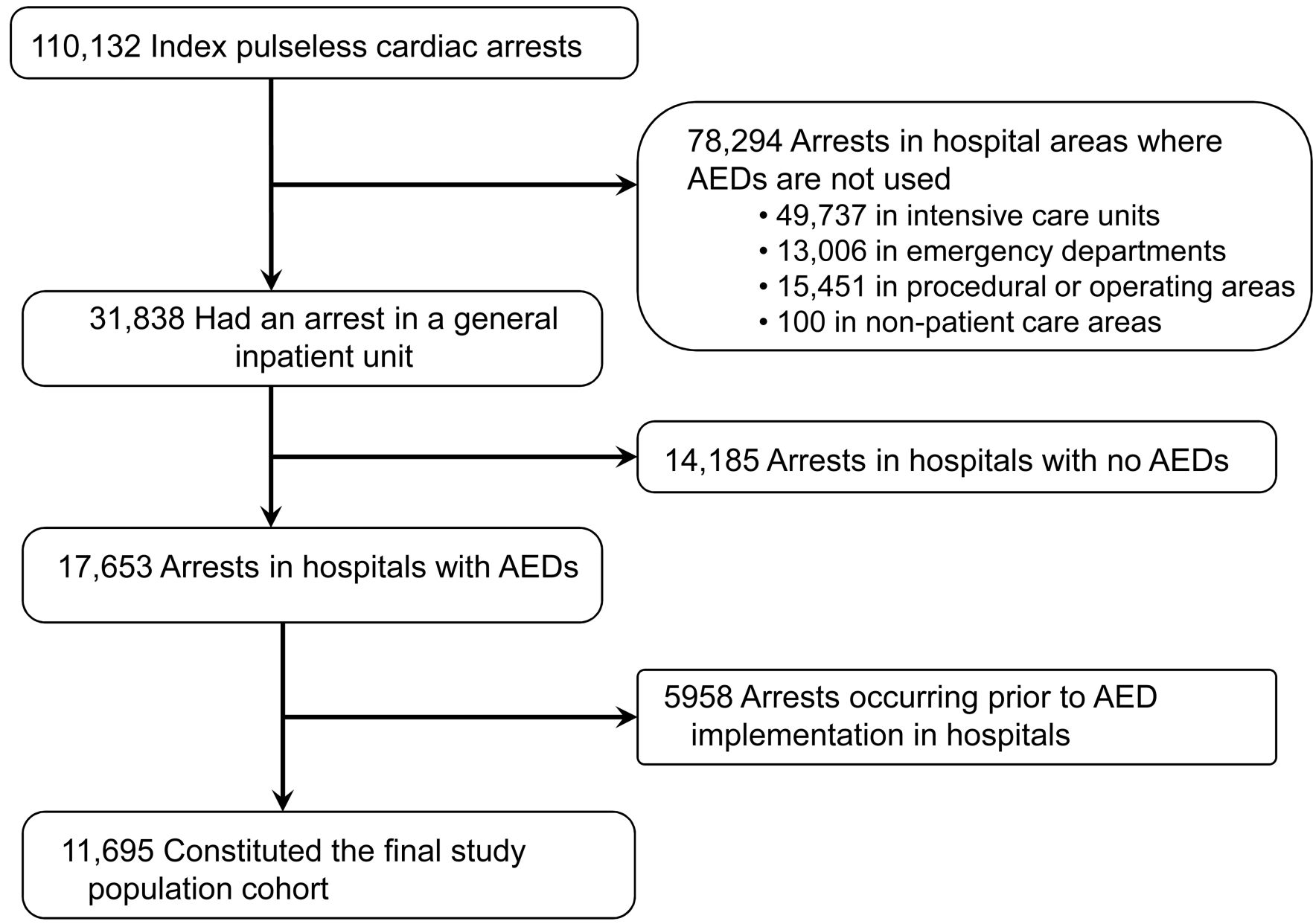

Figure 1. Study Cohort

Of the initial 110,132 index cases of in-hospital cardiac arrest in the National Registry of Cardiopulmonary Resuscitation, 11,695 eligible patients were included in the final study population. 
Table 1

Patient Characteristics According to AED Use

\begin{tabular}{|c|c|c|c|c|}
\hline & $\begin{array}{c}\text { All } \\
\text { Patients } \\
\mathbf{N}=\mathbf{1 1 , 6 9 5}\end{array}$ & $\begin{array}{c}\text { AED } \\
\text { Used } \\
\mathbf{n}=4515\end{array}$ & $\begin{array}{c}\text { AED } \\
\text { Not Used } \\
n=7180\end{array}$ & P-value \\
\hline \multicolumn{5}{|l|}{ DEMOGRAPHICS } \\
\hline Age, years $\pm \mathrm{SD}$ & $68.6 \pm 15.0$ & $68.5 \pm 14.9$ & $68.7 \pm 15.1$ & 0.49 \\
\hline Sex, no. $(\%)$ & & & & 0.15 \\
\hline Male & $6722(57.5)$ & $2633(58.3)$ & $4089(56.9)$ & \\
\hline Female & $4973(42.5)$ & $1882(41.7)$ & $3091(43.1)$ & \\
\hline Race, no. (\%) & & & & 0.02 \\
\hline White & $7911(67.6)$ & $3037(67.3)$ & $4874(67.9)$ & \\
\hline Black & $2741(23.4)$ & $1108(24.5)$ & $1633(22.7)$ & \\
\hline Other/Unknown & $1043(8.9)$ & $370(8.2)$ & $673(9.4)$ & \\
\hline \multicolumn{5}{|c|}{ CONDITIONS PRIOR TO ARREST, no. (\%) } \\
\hline \multicolumn{5}{|l|}{ Cardiac } \\
\hline Arrhythmia & $3727(31.9)$ & $1422(31.5)$ & $2305(32.1)$ & 0.49 \\
\hline History of heart failure & $2878(24.6)$ & $1084(24.0)$ & $1794(25.0)$ & 0.23 \\
\hline Heart failure this admission & $2374(20.3)$ & $926(20.5)$ & $1448(20.2)$ & 0.65 \\
\hline History of myocardial infarction & $2048(17.5)$ & $770(17.1)$ & $1278(17.8)$ & 0.30 \\
\hline Myocardial infarction this admission & $1382(11.8)$ & $519(11.5)$ & $863(12.0)$ & 0.39 \\
\hline \multicolumn{5}{|l|}{ Non-cardiac } \\
\hline Respiratory insufficiency & $3948(33.8)$ & $1448(32.1)$ & $2500(34.8)$ & 0.002 \\
\hline Diabetes mellitus & $3938(33.7)$ & $1558(34.5)$ & $2380(33.1)$ & 0.13 \\
\hline Renal insufficiency & $3740(32.0)$ & $1482(32.8)$ & $2258(31.4)$ & 0.12 \\
\hline Metastatic/hematologic malignancy & $1855(15.9)$ & $770(17.1)$ & $1085(15.1)$ & 0.01 \\
\hline Hypotension/hypoperfusion & $1767(15.1)$ & $648(14.4)$ & $1119(15.6)$ & 0.07 \\
\hline Pneumonia & $1686(14.4)$ & $629(13.9)$ & $1057(14.7)$ & 0.24 \\
\hline Baseline depression in CNS function & $1648(14.1)$ & $686(15.2)$ & $962(13.4)$ & 0.01 \\
\hline Metabolic/electrolyte abnormality & $1527(13.1)$ & $625(13.8)$ & $902(12.6)$ & 0.05 \\
\hline Septicemia & $1490(12.7)$ & $562(12.4)$ & $928(12.9)$ & 0.45 \\
\hline Acute CNS non-stroke event & $925(7.9)$ & $332(7.4)$ & $593(8.3)$ & 0.08 \\
\hline Hepatic insufficiency & $740(6.3)$ & $293(6.5)$ & $447(6.2)$ & 0.57 \\
\hline Acute stroke & $493(4.2)$ & $192(4.3)$ & $301(4.2)$ & 0.87 \\
\hline Major trauma & $201(1.7)$ & $68(1.5)$ & $133(1.9)$ & 0.16 \\
\hline
\end{tabular}

Abbreviations: CNS, central nervous system; SD, standard deviation 
Table 2

Arrest and Hospital Characteristics According to AED Use

\begin{tabular}{|c|c|c|c|c|}
\hline & $\begin{array}{c}\text { All } \\
\text { Patients } \\
\mathbf{N}=\mathbf{1 1 , 6 9 5}\end{array}$ & $\begin{array}{c}\text { AED } \\
\text { Used } \\
\mathbf{n}=4515\end{array}$ & $\begin{array}{c}\text { AED } \\
\text { Not Used } \\
\text { n=7180 }\end{array}$ & P-value \\
\hline \multicolumn{5}{|c|}{ CHARACTERISTICS OF ARREST, no. (\%) } \\
\hline Monitoring Status & & & & $<.001$ \\
\hline Monitored unit & $6260(53.5)$ & $2104(46.6)$ & $4156(57.9)$ & \\
\hline Non-monitored unit & $5435(46.5)$ & $2411(53.4)$ & $3024(42.1)$ & \\
\hline Time of day & & & & 0.09 \\
\hline Day $(8 a-5 p)$ & $7180(61.4)$ & $2821(62.5 \%)$ & $4359(60.7 \%)$ & \\
\hline Night & $4515(38.6)$ & $1694(37.5 \%)$ & $2821(39.3 \%)$ & \\
\hline Time of week & & & & 0.51 \\
\hline Weekday & $7880(67.4)$ & $3026(67.0)$ & $485467.6)$ & \\
\hline Weekend & $3815(32.6)$ & $1489(33.0)$ & $2326(32.4)$ & \\
\hline Initial rhythm & & & & $<.001$ \\
\hline Asystole & $5094(43.6)$ & $2039(45.2)$ & $3055(42.5)$ & \\
\hline Pulseless electrical activity & $4522(38.7)$ & $1529(33.9)$ & $2993(41.7)$ & \\
\hline Ventricular fibrillation & $1369(11.7)$ & $619(13.7)$ & $750(10.4)$ & \\
\hline Pulseless ventricular tachycardia & $710(6.1)$ & $328(7.3)$ & $382(5.3)$ & \\
\hline Hospital-wide code blue called & $11,387(97.4)$ & $4413(97.7)$ & $6974(97.1)$ & 0.95 \\
\hline \multicolumn{5}{|c|}{ HOSPITAL CHARACTERISTICS, no. (\%) } \\
\hline Hospital size & & & & $<0.001$ \\
\hline$<250$ beds & $1996(17.1)$ & $1061(23.5)$ & $935(13.0)$ & \\
\hline $250-499$ beds & $4168(35.6)$ & $1490(33.0)$ & $2678(37.3)$ & \\
\hline 2500 beds & $5531(47.3)$ & $1964(43.5)$ & $3567(49.7)$ & \\
\hline Geographic region & & & & $<0.001$ \\
\hline Northeast & $1598(13.7)$ & $492(10.9)$ & $1106(15.4)$ & \\
\hline Midwest & $2936(25.1)$ & $1170(25.9)$ & $1766(24.6)$ & \\
\hline South & $5354(45.8)$ & $2203(48.8)$ & $3151(43.9)$ & \\
\hline West & $1807(15.5)$ & $650(14.4)$ & $1157(16.1)$ & \\
\hline Teaching status & & & & 0.61 \\
\hline None & $4410(37.7)$ & $1689(37.4)$ & $2721(37.9)$ & \\
\hline Residency program & $7285(62.3)$ & $2826(62.6)$ & $4459(62.1)$ & \\
\hline
\end{tabular}




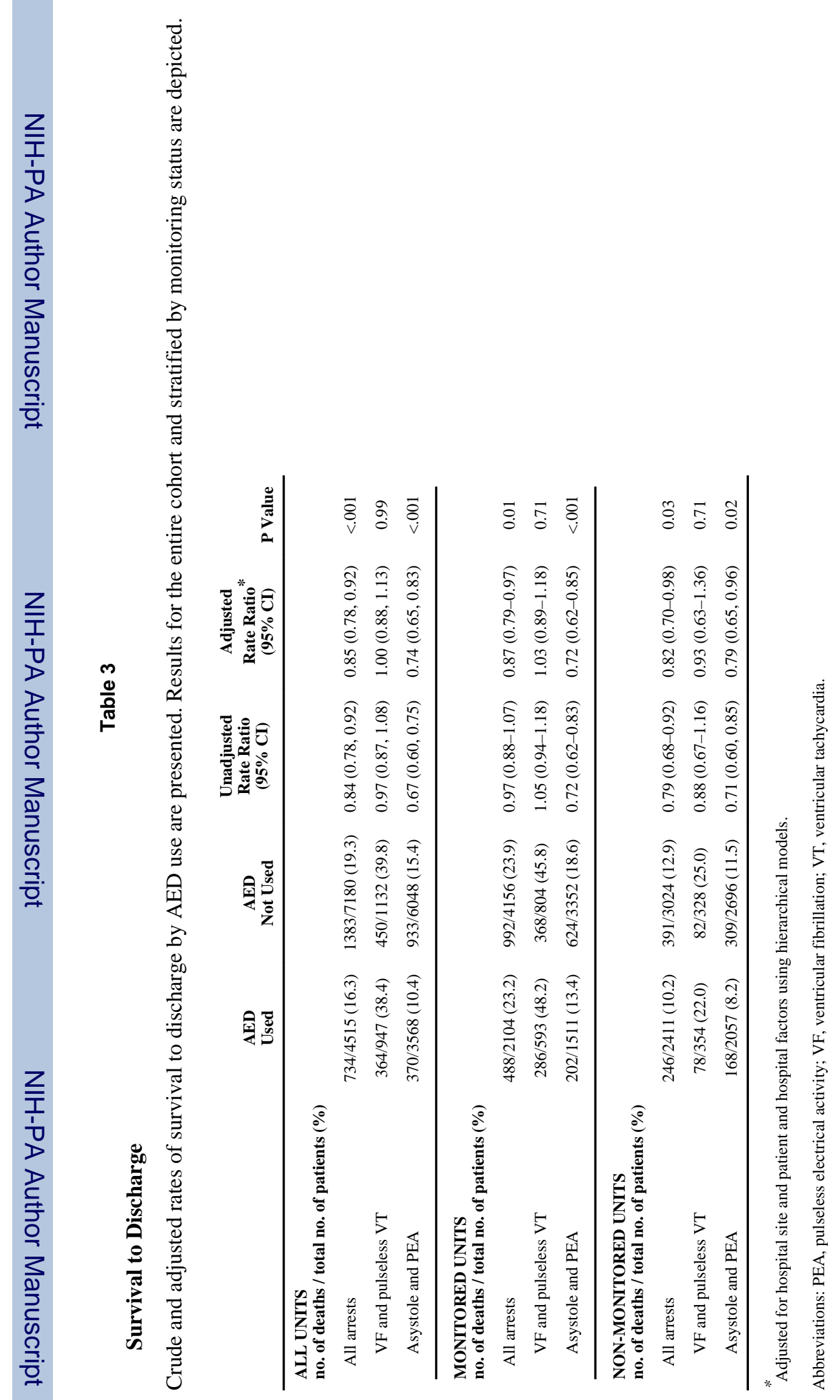

JAMA. Author manuscript; available in PMC 2013 March 05. 


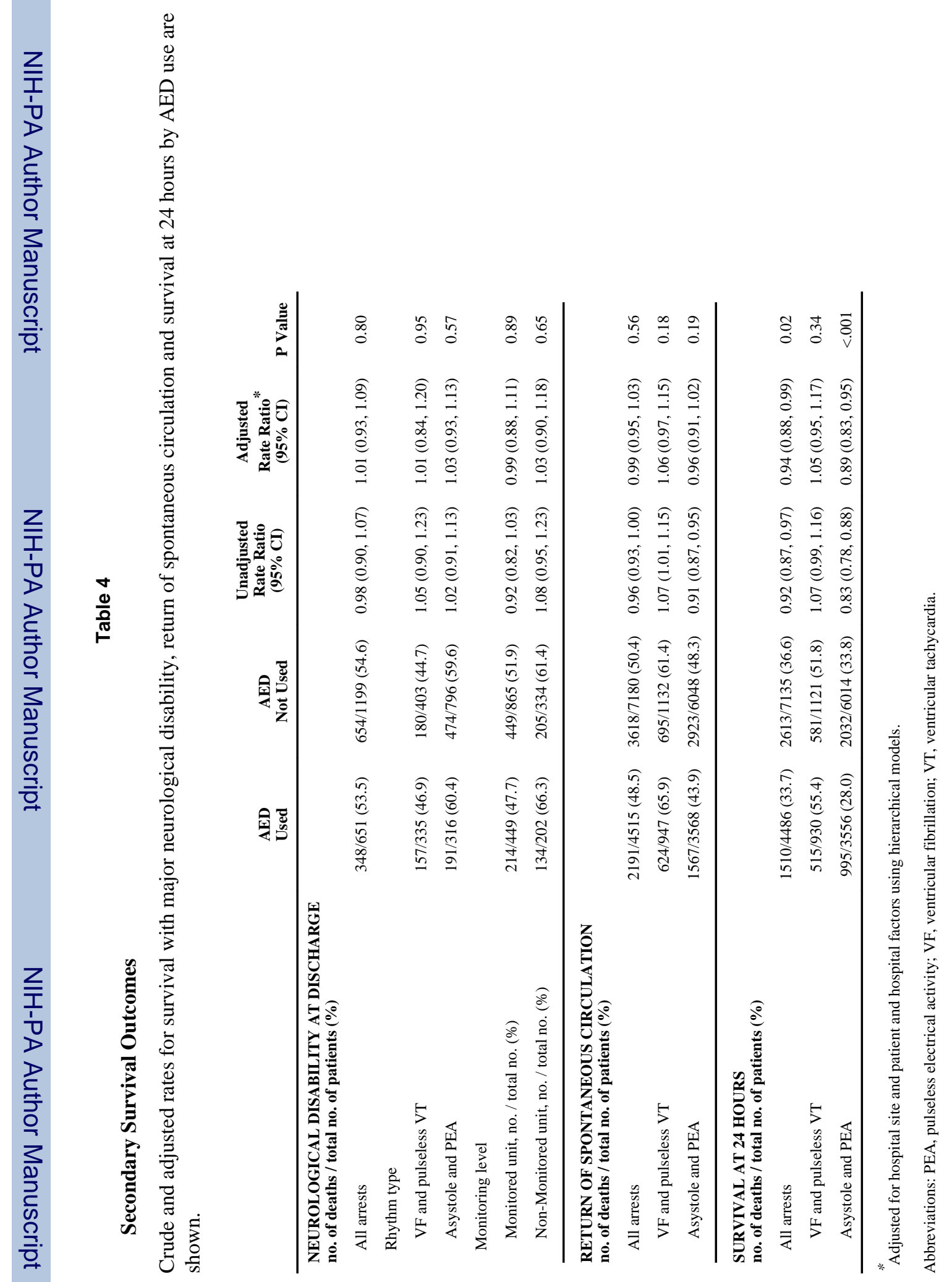

